\title{
Review of: "Conserved topology of virus glycoepitopes presents novel targets for repurposing HIV antibody 2G12"
}

\author{
Barry O'Keefe ${ }^{1}$ \\ 1 National Institutes of Health
}

Potential competing interests: The author(s) declared that no potential competing interests exist.

The manuscript entitled "Conserved topology of virus glycoepitopes presents novel targets for repurposing HIV antibody 2G12" by Miller et al. describes a set of experiments assessing the activity of the antibody 2G12 in the context of influenza (e.g. binding, antiviral activity). The authors use a variety of analysis of publicly-available structural information to draw conclusions on structure-activity relationships that they then bring together into a "predictive" model for assessing potential viral susceptibility to $2 \mathrm{G} 12$. Unfortunately, for several major reasons, the data provided do not justify the conclusions drawn by the authors and therefore this reviewer recommends that this manuscript be rejected.

Below are several specific points that have led me to this conclusion:

1. The actual data presented in this manuscript is minimal. It is basically 1 ELISA experiment on 2 G12 binding to recombinant HA, 1 hemagglutination study, and 1 set of contract anti-influenza activity results reporting $\mathrm{IC}_{50}$ data for several influenza viruses (Note: the authors mix freely between units of ug/ml for antiviral activity and molar measurements for binding, even in the same sentence, not acceptable). This is very little actual data to base the broad conclusions the authors report. Several other important data pieces have been left out such as: a) source of the HA proteins, b) gycosylation on the HA proteins used in the experiments (not those previously published from other sources), c)acknowledgement of the differences between $\mathrm{N}$-linked glycans (complex vs. hybrid vs. high mannose) and data showing presence or absence of each on the specific HA proteins studied, d) specific binding data not based on an ELISA experiment which is a relative and not direct measurement of binding - SPR or ITC binding data would be much more reliable and directly comparable to the literature, e) experimental proof of the quality of their predictive model from additional antiviral tests against viruses identified by their model.

2. The authors make a variety of assumptions based upon literature reports that are not justified. For example, potential N-glycan attachment sites taken from sequence data do not mean that those sites bear glycans that will bind $2 \mathrm{G} 12$, in fact most will not. The authors ignore the copious literature on the specific glycosylation patters of influenza HA. This diminishes the importance of their conclusions. 
3. Though the authors make assertions of the importance of two glycan binding sites with which $2 \mathrm{G} 12$ can simultaneously engage on the susceptibility of specific influenza strains, they completely ignore the published literature on antiviral lectins which has extensively dealt with the importance of cross-linking high-mannose oligosaccharides for optimal antiviral efficacy. They also ignore the published literature on the changes in glycosylation that occur on influenza HA as influenza strains are adapted for lethality in mice. Several of the strains which they have chosen for this manuscript have been so adapted and so, their glycosylation patterns have changed.

4. The antiviral data shown (only for 4 viral strains) has different inoculum PFUs for the viruses, this could skew the resulting antiviral EC50 data making the authors conclusions questionable.

5. The authors freely mix binding data on some viral HAs with hemagglutination data on other Has with antiviral data on still other viral strains and infer that it is directly comparable. This is not so. Figure 2 purports to draw structural conclusions from this mix of data. Such conclusions cannot be drawn from such variant data sets. They further base their structural model on just 2 PDB structures ( one from HIV gp120 and one from SARS-CoV-2 Spike, none from influenza HA) and then "combine them with functional data from experimental binding and neutralization assays"-(a single paper and the very limited data presented in Figure 1 for their influenza-related structural information). They use another single manuscript reference to justify calling certain oligosaccharide attachment sites "high mannose" without any direct evidence. they also take the structural rationale described in that paper as the only reason for high-mannose oligosaccharides to be present on envelope glycoproteins which is just not correct. Such structural speculation in the absence of much mor direct experimental data on specific glycans and directly comparable binding data is not supportable.

6. The purported "validation" of the proposed topological glycan epitope model shown in Figure 3 is not scientifically justified. There are many things wrong. First, the authors suddenly include yeast in their model, why? Yeast is known to produce predominantly high mannose oligosaccharides which would bind 2G12. the inclusion of yeast skews the results of the regression significantly. they should not be included. Second, even with the yeast included, the $\mathrm{R}^{2}$ of 0.6 is not convincingly significant by any measure. Third, they state that the model is correlating the $2 \mathrm{G} 12$ binding strength with their model score but use disparate measure of binding (ELISA, antiviral activity, only single HIV and SARS-CoV-2 data from binding methods other than those used in this paper). Overall, just not acceptable.

Overall the manuscript details few experiments from which, together with literature reports, the authors draw unjustified conclusions and speculate without sufficient support. They then base a model on this speculation and provide a very weak validation of that model. I cannot therefore recommend that this 
manuscript be published and recommend its rejection by Scientific Reports. 